\title{
Crease Enhancement Diffusion
}

\author{
Andres Fco. Solé ${ }^{1}$, Antonio López ${ }^{\dagger}$ and Guillermo Sapiro ${ }^{\ddagger 2}$ \\ ${ }^{\dagger}$ Centre de Visió per Computador, Universitat Autonoma de Barcelona, Edifici O, Spain ; \\ and ${ }^{\ddagger}$ University of Minnesota, Department of Electrical and Computer Engineering
}

Ridge and valley structures are important image features, specially in oriented textures. Usually, the extraction of these structures requires a prefiltering step to regularize the source image. In this paper, we show that classical diffusion based filters are not appropriate for this task, and propose a new filtering process. This new filter can be tuned to join broken valley or ridge lines while preserving their junctions, thus preserving the general topology of the lines obtained afterwards. This filter can be interpreted as an example of the intrinsic introduction of structure on diffusion processes. Keywords: diffusion process, structure tensor, ridges, valleys, anisotropic diffusion, PDE's.

\section{INTRODUCTION}

Ridge and valley structures are important linear image features, specially for highly oriented textures. Usually, the extraction of these structures requires a prfiltering step to regularize the original image. There are many works devoted to the extraction of ridge/valley structures $[3,8,9]$. In any case, some kind of regularization of the input image is needed to obtain stable and meaningful results. Frequently, this regularization consists simply of a convolution by a Gaussian kernel. In case of highly oriented textures, this approach is unsuitable because the inner structures can be destroyed. A solution is to use non isotropic filters, like the proposed by Perona and Malik [10,11]. However, it has proved to be more appropriated the use of an explicit directed diffusion process. A recent approach in this context has been provided by Weickert $[12,13,14]$. In his work, he proposes a new diffusion process based on the structure tensor, which provides a local description of the anisotropy in a neighborhood of the image. This new diffusion process has two important properties: it makes possible to close some interrupted linear structures and enhances reliable linear structures in the input image. On

\footnotetext{
${ }^{1}$ This work was partially supported by a grant from the Direcció General de Recerca de Catalunya, BE1999

${ }^{2}$ This work was partially supported by a grant from the Office of Naval Research ONR-N0001497-1-0509, the Office of Naval Research Young Investigator Award, the Presidential Early Career Awards for Scientists and Engineers (PECASE), a National Science Foundation CAREER Award, and by the National Science Foundation Learning and Intelligent Systems Program (LIS).
} 
the other hand, an undesirable effect of this diffusion process is that ridge/valley junctions are destroyed and non linear structures are deformed. In this paper we present a new diffusion process that enhances ridge/valley structures. This process is based on a new diffusion tensor that includes the differential structure of ridges and valleys in an image. This tensor, that we call creaseness tensor, provides the creaseness direction in each point and a local measure of creaseness. In Sect. 2 we review what is a diffusion process. In Sect. 3 we define the creaseness tensor and construct the corresponding diffusion process. Section 4 presents comparative results. Finally, Sect. 5 summarizes the main conclusions.

\section{DIFFUSION PROCESSES}

Diffusion processes derive from Fick's law [5] and the continuity equation. Fick's law expresses that a gradient concentration leads to a flow which compensates it. If we include Fick's law into the continuity equation, which expresses that mass is only transported but can neither be created nor destroyed, we obtain the diffusion equation:

$$
\frac{\partial u}{\partial t}=\operatorname{div}(D \cdot \nabla u)
$$

where $D$ is a diffusion tensor and $u$ corresponds to the mass concentration. The diffusion tensor define the diffusion process, for example, if we choose $D$ as the identity matrix we have a particular case of the diffusion equation, the heat equation. This special case can analytically be solved and one can prove that it corresponds to a convolution of the initial function $u$ with a Gaussian kernel. If we think of $u$ as the grey level of an image $I$, we obtain the Gaussian smoothing as a special case of the diffusion equation $[7,15]$. This diffusion process suffers from the well known problem of displacement of edges due to Gaussian blurring. In order to solve this problem, Perona and Malik proposed another diffusion process [10] (see also [11]) which can be seen as a special case of the diffusion equation:

$$
\frac{\partial I}{\partial t}=\operatorname{div}\left(\frac{1}{1+\frac{\|\nabla I\|^{2}}{K^{2}}} \cdot \nabla I\right)
$$

where $K$ is a parameter controlling the diffusion strength. However, neither the Gaussian diffusion nor the Perona-Malik's are tuned to enhance linear structures while regularizing the image. In these processes the diffusion directions are always collinear to the image gradient $\nabla I$ and its perpendicular $\nabla I^{\perp}$. Then, with the purpose of enhancing linear structures, Weickert proposed in [14] the tuning of the diffusion directions according to the dominant orientation at each image pixel. This dominant orientation at each pixel is obtained through the structure tensor analysis [6]. The structure tensor can be defined as the convolution of the Gaussian kernel with the tensor product of the regularized gradient by itself:

$$
S_{\rho, \sigma}=G_{\rho} *\left(\nabla I_{\sigma} \nabla I_{\sigma}^{T}\right)
$$

The parameter $\sigma$ is the differentiation scale and controls the size of the objects whose orientation has to be determined, while $\rho$ is the integration scale and controls the size of the neighborhood in which an orientation is dominant. Weickert 
proposed a diffusion process based on the construction of a diffusion tensor whose eigendirections coincide with the eigenvectors of the structure tensor, but having different eigenvalues, namely:

$$
\left\{\begin{array}{l}
\lambda_{1}=\alpha \\
\lambda_{2}=\alpha+(1-\alpha) \exp \left(\frac{-C}{\left(\mu_{1}-\mu_{2}\right)^{2 m}}\right)
\end{array}\right.
$$

where $\mu_{1}$ and $\mu_{2}$ are the eigenvalues of the structure tensor and $m, C$ and $\alpha$ are parameters controlling the exponential shape and the diffusion strength in the non coherence direction. Since this diffusion process enhances coherent flow-like structures it seems appropriate for regularizing the image before extracting ridges and valleys. However, we have observed that the inner structures of ridges and valleys are modified by this filtering process. Specially, many junctions disappear and original structures are deformed. In order to try to solve these problems we propose a new diffusion process that introduces information about the crease structure of the image.

\section{CREASE DIFFUSION FLOW}

In this section we propose a new diffusion process that smoothes the original image while preserving and enhancing the intrinsic crease structure. First, we make a brief review of the classical theory surrounding the problem of surface structure classification, giving an intuitive and geometric interpretation of it. Then we make use of this classical theory in order to define a new diffusion tensor, that we call crease diffusion tensor.

\subsection{Local surface classification}

Consider a gray level image $I(x, y)$ as a surface graph. Then we can describe this image locally by means of its Taylor expansion. In fact, if we are only interested in the shape of the image we only need the second order derivatives information, that is the Hessian of $I$. The Hessian provides us an analytical local representation of a surface $z=u(x, y)$ in each point $p=\left(x_{0}, y_{0}\right)$. The corresponding eigenvectors and eigenvalues, that we denote as $v_{1}, v_{2}, h_{1}$ and $h_{2}$ respectively, can be used to make the classification presented in Fig. 1 (left) in terms of surface local structure. In figure 1 (left) we can see the local surface classification obtained using only the Hessian information. But there exists a more accurate definition for ridges and valleys using also the gradient information. That is, we consider a point to be a ridge/valley point if the gradient is orthogonal to the eigenvector corresponding to the greatest eigenvalue of the Hessian. In addition, the following relationship between the surface principal curvatures $\left(k_{i}\right)$ and Hessian eigenvalues $\left(h_{i}\right)$ holds:

$$
k_{i}=\frac{h_{i}}{\sqrt{1+\|\nabla u\|^{2}}} \text { if } \nabla u \cdot v_{i}=0
$$

That is, in the case of ridges and valleys the eigendirection $v_{i}$ and the normalized eigenvalue $\left(k_{i}\right)$ coincide exactly with the principal direction and the maximum principal curvature respectively. We can obtain directly this normalized eigenvalues for the Hessian if we analyze the normalized Hessian below instead of the classical 

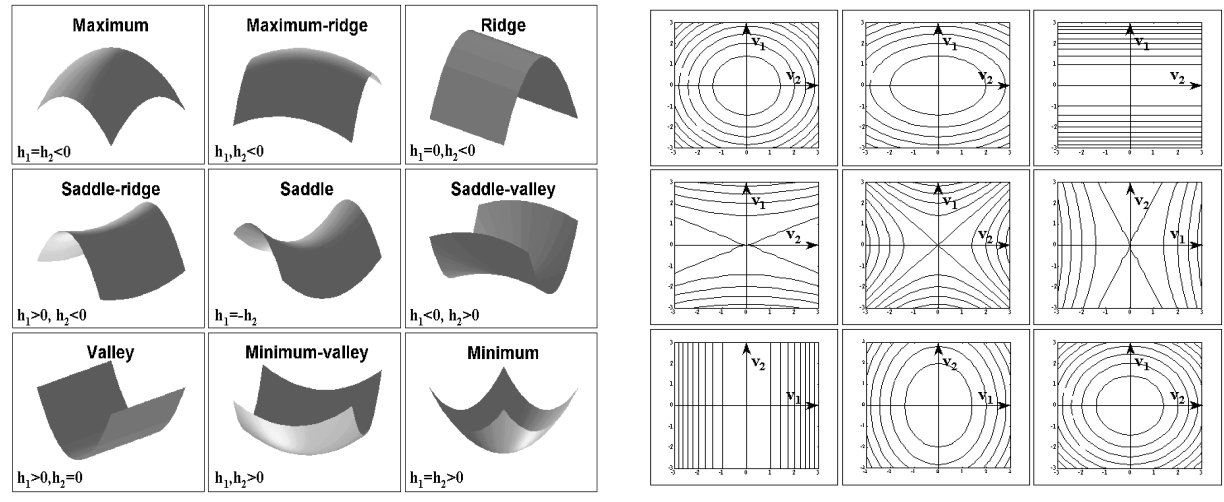

FIG. 1. Left: Surfaces obtained varying the $h_{1}$ and $h_{2}$ eigenvalues of a quadratic function. Right: Level sets corresponding to the surfaces on the left and its eigendirections corresponding to $\bar{k}_{1}$ and $\bar{k}_{2}$ respectively.

one,

$$
\frac{1}{\sqrt{1+\|\nabla u\|^{2}}}\left(\begin{array}{cc}
\frac{\partial^{2} u}{\partial x^{2}} & \frac{\partial^{2} u}{\partial x \partial y} \\
\frac{\partial^{2} u}{\partial x \partial y} & \frac{\partial^{2} u}{\partial y^{2}}
\end{array}\right)
$$

Unfortunately, for our purposes the classification provided in Fig. 1 (left) is not adequate for two main reasons. The first reason is that we are looking for a positive valued descriptor which reaches its highest value in the presence of ridges or valleys. On the other hand we also need a bounded descriptor. In order to achieve these requirements we propose the following shape descriptor:

$$
\mu=\frac{\tilde{k}_{1}-\tilde{k}_{2}}{\tilde{k}_{1}+\tilde{k}_{2}}
$$

where $\tilde{k}_{1}=\max \left(\left|k_{1}\right|,\left|k_{2}\right|\right)$ and $\tilde{k}_{2}=\min \left(\left|k_{1}\right|,\left|k_{2}\right|\right)$. It is easy to prove that $\mu$ is bounded, concretely $0 \leq \mu \leq 1$. Since we are interested in ridges and valleys separately we can consider the following factorization of the descriptor $\mu$ :

$$
\mu_{r}=\left\{\begin{array}{ll}
\frac{\tilde{k}_{1}-\tilde{k}_{2}}{\tilde{k}_{1}+\tilde{k}_{2}} & \text { if } \quad k_{1}<0 \\
0 & \text { if } \quad k_{1} \geq 0
\end{array} \quad \mu_{v}=\left\{\begin{array}{lll}
\frac{\tilde{k}_{1}-\tilde{k}_{2}}{\tilde{k}_{1}+\tilde{k}_{2}} & \text { if } & k_{1}>0 \\
0 & \text { if } & k_{1} \leq 0
\end{array}\right.\right.
$$

where $\mu_{r}$ and $\mu_{v}$ reaches its highest values in the presence of ridges and valleys respectively. In fact we obtain the classification of the Table 1 (note that $\mu=$ $\left.\mu_{r}+\mu_{v}\right)$. The direction associated with the largest eigenvalue (in absolute value) corresponds to the local crease direction of the surface. In Figure 1 (right) we can see the crease direction (labeled as $v_{2}$ ).

\subsection{The Creaseness Diffusion Tensor}

In order to construct a multilocal representation of the normalized Hessian (6) we can make a double regularization step as in the case of the structure tensor (see 
TABLE 1

Different surfaces grouped in function of $\tilde{\boldsymbol{k}}_{1}, \tilde{\boldsymbol{k}}_{2}$.

\begin{tabular}{|c|c|c|}
\hline $\begin{array}{l}\tilde{k}_{1}>0 \\
\tilde{k}_{2}=0\end{array}$ & $\begin{array}{l}\text { Ridge } \\
\text { Valley }\end{array}$ & $\mu=1$ \\
\hline$\tilde{k}_{1}>\tilde{k}_{2}$ & $\begin{array}{l}\text { Saddle-Valley } \\
\text { Saddle-Ridge } \\
\text { Maximum-Ridge } \\
\text { Minimum-Valley }\end{array}$ & $0<\mu<1$ \\
\hline$\tilde{k}_{1}=\tilde{k}_{2}$ & $\begin{array}{l}\text { Minimum } \\
\text { Maximum } \\
\text { Perfect Saddle }\end{array}$ & $\mu=0$ \\
\hline
\end{tabular}

eq. (9)). First we regularize the initial image $u$ in order to obtain robust derivatives (derivation step) and then regularize the tensor field (integration step):

$$
G_{\rho} *\left[\frac{1}{\sqrt{1+\left\|\nabla u_{\sigma}\right\|^{2}}}\left(\begin{array}{cc}
\frac{\partial^{2} u_{\sigma}}{\partial x^{2}} & \frac{\partial^{2} u_{\sigma}}{\partial x \partial y} \\
\frac{\partial^{2} u_{\sigma}}{\partial x \partial y} & \frac{\partial^{2} u_{\sigma}}{\partial y^{2}}
\end{array}\right)\right]
$$

where $G_{\rho}$ is a Gaussian kernel of size $\rho$. This tensor provides a multilocal version of the eigendirections and normalized eigenvalues of the Hessian. We denote as $v_{1}$ the first eigendirection corresponding to the highest eigenvalue in absolute value $\left(\tilde{k}_{1}\right)$ and $v_{2}$ the one corresponding to the lowest. The creaseness diffusion tensor is constructed in order to have the same eigenvectors $v_{1}$ and $v_{2}$ and the following associated eigenvalues:

$$
\left\{\begin{array}{l}
\lambda_{1}=\epsilon \quad \epsilon \in(0,1) \\
\lambda_{2}=\alpha \mu_{r}+\beta \mu_{v} \quad \alpha, \beta \in[0,1]
\end{array}\right.
$$

where $\alpha$ and $\beta$ are parameters controlling the diffusion strength in the presence of ridges and valleys respectively and $\epsilon$ controls the diffusion in the direction perpendicular to the creaseness direction and assures the semidefinite property of the diffusion tensor. Due to the regularization steps, the diffusion process which we propose here is well posed and the existence and uniqueness of solutions can be proved in the same way as in $[2,4]$. Interesting values for the couple $(\alpha, \beta)$ are $(1,0)$, pure ridge diffusion, $(0,1)$ pure valley diffusion and $(1,1)$ ridge-valley diffusion.

\section{RESULTS}

Figure 2 shows the behavior of our filter both in a synthetic image and on a real fingerprint image. The synthetic image (Fig. 2 left)is formed by valley (darker) and ridge (brighter) structures. In the central part the valleys and ridge are more radial while as we move away from the center the crease structure become more like concentric ellipses. The original image (top left) was filtered using our proposal (left bottom) and their valleys were extracted (right hand of each image). We can observe that the filtering process (which is tuned to enhance mainly the valley structures) enhances the valleys of the original image leading to a better results on the valley extraction. We have performed the same experiment in the case of the fingerprint. One can also notice that the valley structure was enhanced leading to a 

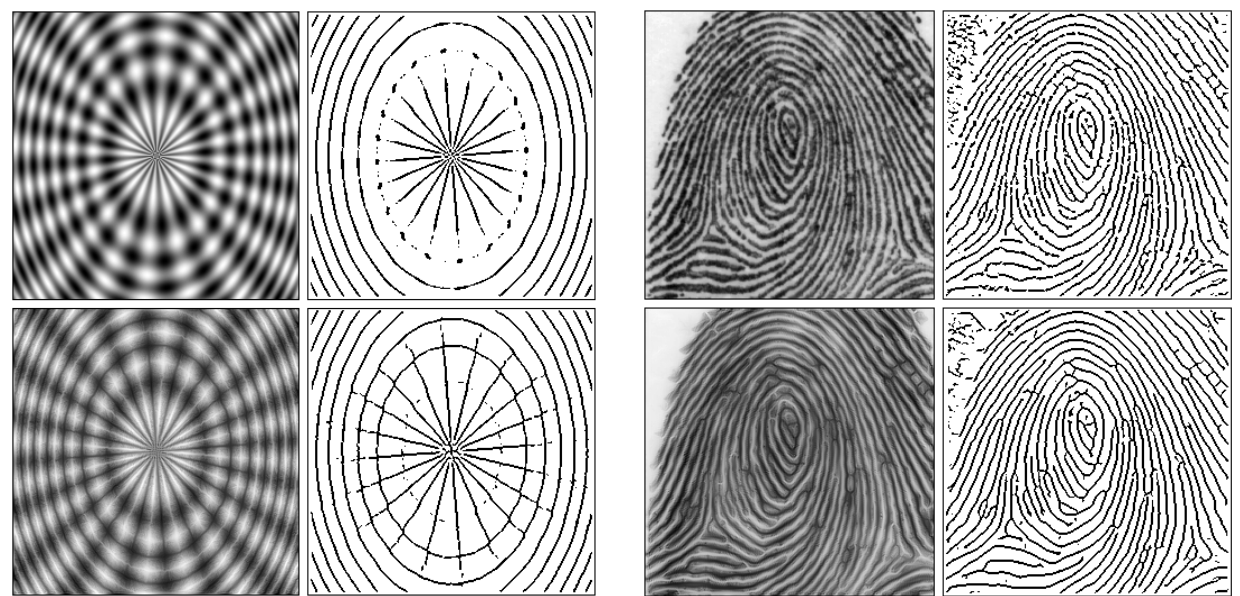

FIG. 2. Left: Parameters $\alpha=1 \beta=0.5 \sigma=1 \rho=0.5$. Right: Parameters $\alpha=1$ $\beta=1 \sigma=1 \rho=0.5$.

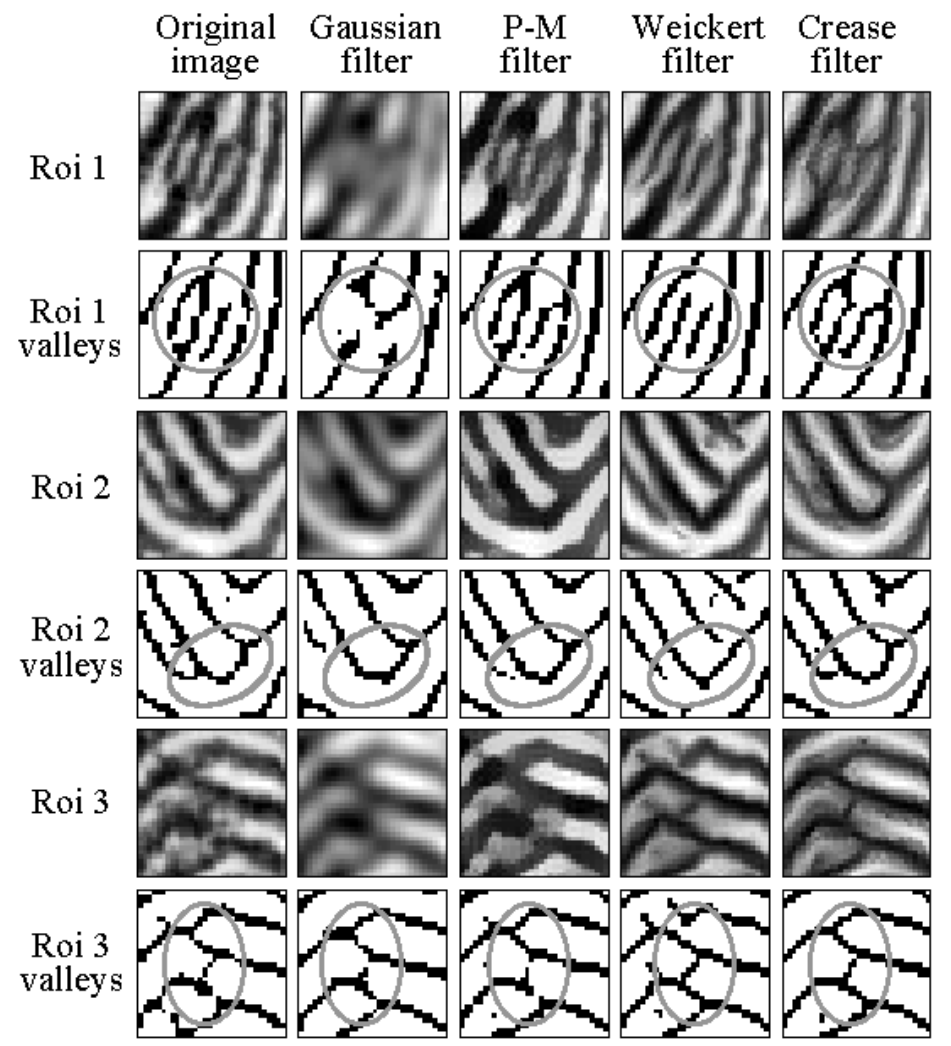

FIG. 3. ROIs from the fingerprint image (Fig. 2 right side). Each ROI was filtered using Gaussian smoothing (second column, $\sigma=2$ ), Perona-Malik's smoothing (third column, $K=4$, 10 iterations), Weickert's smoothing (fourth column, $\sigma=1, \rho=$ 1, 10 iterations) and finally our filter (fifth column, $\sigma=1, \rho=1, \alpha=1, \beta=0.6,10$ iterations). 
better valley extraction. The fingerprint is analyzed more precisely in the following figure.

In Fig. 3 we zoom three regions of interest (ROIs of $32 \times 32$ pixels) from the fingerprint image (Fig. 2 right). Each ROI was filtered using Gaussian smoothing (second column), Perona-Malik's smoothing (third column), Weickert's smoothing (fourth column) and finally our filter (fifth column). The corresponding valleys (row below each ROI) were also computed in order to compare the effects of the filtering step. The junctions in each valley image are surrounded with a gray oval in order to make easy their localization. Although the structures are more preserved in the case of the Perona-Malik smoothing than in the case of Gaussian smoothing we can observe that some linear structures and also some junctions are destroyed. Weickert's filter preserves much more the linear information but also the topology (junctions) are destroyed. In our case we observe a better behavior. Linear structures are enhanced while preserving its own topology.

\section{DISCUSSION}

We have presented a new diffusion process to enhance ridge/valley structures, that we term crease enhancement diffusion. It is based on two main ideas: a directed diffusion process and a stable bounded creaseness operator. The advantages of this filtering process are that while crease features are enhanced, artifacts in the image do not appear, contours are preserved and junctions are not interrupted. We have compared it with other classical filters like Gaussian or Perona-Malik smoothing, showing their problems, specially in the case of highly oriented textures. Also we have compared it with a recent approach proposed by Weickert, the coherence enhancement diffusion, from which we have borrow the idea of coherence. We have shown that although Weickert's filter enhances linear structures (like ridges and valleys) many junctions disappear and some artifacts appear, that is, the crease structure is not preserved. Our filtering process avoids these problems to a large extent. This new filter should be also interpreted as an example of introducing the enhancement of specific geometric structures in diffusion processes. This is done directly in the diffusion process, and not explicitly favored via outliers as in [1].

\section{ACKNOWLEDGMENT}

We would like to thank Professor Vicent Casselles from the Universitat Pompeu Fabre for useful comments during the development of this work.

\section{REFERENCES}

1. M Black, G Sapiro, D Marimont, and D Heeger. Robust anisotropic diffusion. IEEE Trans. Image Processin, 73:421-432, 1998.

2. Vicent Casselles and Leonid Rudin. Multiscale total variation. Workshop on Image Processing, Palma, Spain, 1995.

3. D. Eberly, R. Gardner, B. Morse, S. Pizer, and C. Scharlach. Ridges for image analysis. J. of Mathematical Imaging and Vision, 4:353-373, 1994.

4. J. M. Morel F. Catte, P. L. Lions and B. Coll. Image selective smoothing and edge detection by nonlinear diffusion. SIAM J. Numer. Anal.

5. G.L. Flynn. Mass transport phenomena and models: Theoretical concepts. In J. Pharm. Sci., 63 (4), pages 479-510, 1974.

6. B. Jähne. Spatio-temporal image processing, volume 751 of Lecture Notes in Computer Science, chapter 8, pages 143-152. Springer-Verlag, 1993. 
7. JJ Koenderink and AJ van Doorn. Dynamic shape. Biol. Cyber., 53:367-375, 1986.

8. A. López, F. Lumbreras, J. Serrat, and J. Villanueva. Evaluation of methods for ridge and valley detection. IEEE Trans. on Pattern Analysis and Machine Intelligence, 21(4), 1999.

9. J. Maintz, P. van den Elsen, and M. Viergever. Evaluation of ridge seeking operators for multimodality medical image matching. IEEE Trans. on Pattern Analysis and Machine Intelligence, 18:353-365, 1996.

10. P. Perona and J. Malik. Scale-space and edge detection using anisotropic diffusion. IEEE Computer Society Press, Washington, pages 16-22, 1987.

11. L Rudin, S Osher, and E Fatemi. Nonlinear total variation based noise removal algorithms. Physica D, 60:259-268, 1992.

12. J. Weickert. Anisotropic diffusion filters for image processing based quality control. In Seventh European Conference on Mathematics in Industry, pages 355-362, 1994.

13. J. Weickert. Anisotropic Diffusion in Image Processing. $\mathrm{PhD}$ thesis, Universitat Kaiserslautern, 1996.

14. J. Weickert. Anisotropic diffusion in image processing. In Teubner-Verlag, 1998.

15. AP Witkin. Scale-space filtering. Proc. IJCAI, pages 1019-1021, 1983. 